\title{
Prevenção de Colisões em Serviços de Entregas por Drones em Cidades Inteligentes
}

\author{
Fabíola M. C. de Oliveira ${ }^{1,2}$, Luiz F. Bittencourt ${ }^{2}$, Carlos A. Kamienski ${ }^{1}$ \\ ${ }^{1}$ Centro de Matemática, Computação e Cognição - Universidade Federal do ABC (UFABC) \\ Santo André - SP - Brazil \\ ${ }^{2}$ Instituto de Computação - Universidade Estadual de Campinas \\ Campinas, SP \\ \{fabiola.oliveira, bit\}@ic.unicamp.br, cak@ufabc.edu.br
}

\begin{abstract}
The recent advances in unmanned aerial vehicles (UAV), commonly called drones, and the new communication and artificial intelligence technologies make it possible to develop aerial delivery services for the near future. However, such advances in smart city services require strict security and safety standards. Particularly, collision avoidance strategies are needed to guarantee that drones do not collide with each other, with other aerial objects or beings, and numerous obstacles in typical urban settings. This paper proposes a delivery service scenario for smart cities and assesses the number of drones that collide under three different methods for the situation when a drone approaches another one: keeping the original route, taking a random detour and, taking a detour to the right, like in aviation. The results show that the detours can maintain or eliminate the number of collisions for fleets with few drones but increase the number of collisions when more drones are present. We investigate the travel time of the drones to show how each method behaves and the position of the collisions to identify the collision situation. The main conclusion is that naive and straightforward detour approaches do not guarantee collision avoidance, making a case for more sophisticated ones.
\end{abstract}

Resumo. Os recentes avanços em veículos aéreos não tripulados (VANT), usualmente chamados de drones, aliados às novas tecnologias de comunicação e de inteligência artificial, estão possibilitando o desenvolvimento de serviços aéreos de entregas de mercadorias para um futuro próximo. No entanto, para que tais serviços avançados para cidades inteligentes se tornem realidade, é necessário garantir a segurança nas entregas. Particularmente, é necessário garantir que drones não colidam com outros drones, com outros objetos ou seres voadores, assim como com os vários obstáculos típicos de ambientes urbanos. Esse artigo propõe um cenário de serviço de entregas por drones em cidades inteligentes e avalia o número de drones que colidem sob três abordagens diferentes para a situação em que um drone se aproxima de outro: manter a rota original, efetuar um desvio aleatório e efetuar um desvio à direita, como na aviação. Os resultados mostram que os desvios podem manter ou eliminar o número de colisões para frotas com poucos drones, mas aumentam o número de colisões quando a densidade de drones aumenta. Investigamos o tempo de viagem dos drones para mostrar como cada abordagem se comporta e as posições das colisões 
para identificar a situação das colisões. A principal conclusão é que abordagens simples e ingênuas de desvio não garantem a evasão de colisões, indicando que estratégias mais sofisticadas devem ser aplicadas.

\section{Introdução}

As tendências atuais conduzem a um futuro próximo no qual veículos aéreos não tripulados (VANT), também conhecidos como drones, serão pervasivos em áreas urbanas, oferecendo aos cidadãos serviços como entrega de alimentos e encomendas, detecção de incêndio, combate a incêndio e transporte de equipamentos médicos [Sánchez et al. 2020]. Essas aplicações requerem que os drones voem em altitudes baixas, aumentando a possibilidade de colisões com diversos tipos de obstáculos, tais como prédios, árvores, pessoas, pássaros, entre outros, além da colisão entre drones devida à sua alta densidade em áreas urbanas [Hrabar 2011]. Assim, drones necessitam evitar colisões como um primeiro passo fundamental em direção a um sistema multidrones em áreas urbanas [Sánchez et al. 2020]. Em um espaço aéreo tão ocupado, os drones podem necessitar de um conjunto de estratégias de prevenção de colisão para escolher a melhor opção em cada situação, por exemplo, algoritmos locais para serem usados em emergências e algoritmos globais para o planejamento da trajetória [Yasin et al. 2020].

Embora existam diversas estratégias de prevenção de colisão, segundo nosso conhecimento, nenhum trabalho avaliou essas técnicas no cenário de entrega com drones em que há pousos e decolagens e um número maior de drones. Além disso, a maioria dos trabalhos avalia colisões durante o vôo em cruzeiro, em que normalmente não há variação de altura, reduzindo o problema de colisão a duas dimensões e não considerando colisões que podem ocorrer durante o pouso e decolagem ou testando apenas casos em duas dimensões [Yasin et al. 2020, Gageik et al. 2015, Choi and Kim 2020]. Por fim, os resultados da literatura mostram que nenhuma técnica de prevenção de colisão é capaz de evitar todas as colisões, sendo necessário agregar uma ou mais técnicas de prevenção de colisão [Yasin et al. 2020].

Neste trabalho, nós propomos um cenário de logística multi-drones, no qual várias empresas de compras pela Internet, logísticas e de entrega de alimentos entregam pacotes usando drones. Como a prevenção de colisões é fundamental e não existe um patamar inicial do número de colisões nesse cenário, avaliamos o número de colisões nesse cenário a partir de três estratégias. A primeira estratégia não evita colisões para que tenhamos um valor inicial do patamar de colisões. A segunda estratégia toma um desvio aleatório na tentativa de evitar uma colisão e a terceira estratégia toma um desvio à direita, que é uma estratégia inspirada pelas regras vigentes da aviação [Guan et al. 2020]. As duas últimas estratégias são do tipo detecção e prevenção, no qual, uma vez detectada uma possível colisão, o drone pode imediatamente tomar uma ação para evitar a colisão.

Incluímos essas estratégias no simulador UTSim [Al-Mousa et al. 2019] e o instrumentamos para gerar resultados sobre as colisões para diferentes tamanhos de frotas de drones. Em seguida, avaliamos os resultados gerais e específicos sobre a viagem dos drones para explicar as situações de colisão. Os resultados mostram que as duas estratégias utilizadas são capazes de reduzir ou manter o número de colisões para frotas com poucos drones, mas aumentam esse número quando as frotas possuem 10 ou mais drones. As principais contribuições deste trabalho são mostrar que estratégias mais simples au- 
mentam o número de colisões em um cenário com alta densidade de drones e mostrar a proporção dos dois tipos de colisão: colisões em cruzeiro e colisões devidas às tentativas de decolagem e pouso.

Este trabalho está organizado da seguinte maneira: a seção 2 apresenta os trabalhos relacionados nas áreas de prevenção de colisão aérea e simuladores de drones; a seção 3 descreve o cenário proposto; a seção 4 apresenta os detalhes das simulações realizadas e as abordagens de prevenção de colisão utilizadas; a seção 5 discute os resultados; a seção 6 apresenta as limitações do trabalho e a última seção apresenta a conclusão e os trabalhos futuros.

\section{Trabalhos Relacionados}

Nesta seção, discutimos os principais trabalhos que abordam técnicas de prevenção de colisão aérea e simuladores de drones.

\subsection{Técnicas de Prevenção de Colisão Aérea}

O artigo [Yasin et al. 2020] revisa várias estratégias de prevenção de colisão para veículos autônomos, com ênfase em drones, categorizando-as em reativas, que devem responder rapidamente a mudanças no ambiente, ou deliberativas, que utilizam um mapa do ambiente para otimizar uma rota sem colisões. Enquanto as estratégias reativas funcionam em ambientes dinâmicos, as abordagens deliberativas requerem um ambiente estático em que as posições, tamanhos e velocidades de todos os objetos são conhecidos. Assim, as estratégias devem ser combinadas para se ter uma estratégia eficaz e eficiente de prevenção de colisão. As estratégias também foram categorizadas em: geométricas, que utilizam localização e velocidade dos objetos; campos de força, que impõem uma força de atração no destino e uma força de repulsão nos objetos, de forma a se ter um caminho otimizado; otimizadas, que utilizam algoritmos de otimização como algoritmos genéticos e métodos gulosos; e detecção e prevenção, que tomam decisões em tempo de execução quando um objeto é detectado.

O trabalho [Seo et al. 2017] avalia estratégias geométricas e de detecção e prevenção para evitar colisões em vôos em formação com um conjunto de aeronaves. Com o uso de estratégias reativas e locais, os mínimos locais que podem acontecer nas estratégias de campo de força são evitados. Esse trabalho apresenta as características das aeronaves e os raios de detecção e de colisão utilizados. Já o artigo [Lin et al. 2020] evita colisões de aeronaves de asa fixa com múltiplos obstáculos móveis e avalia a prevenção de colisão entre três a seis obstáculos. Sua estratégia modifica a velocidade das aeronaves, considerando que todas as aeronaves viajam a velocidade constante. De acordo com a identificação do número e do tipo de obstáculos (estático ou dinâmico), os autores propõem diferentes estratégias geométricas para prevenção de colisão.

O trabalho [Choi and Kim 2020] propõe uma estratégia baseada em campos de força que funciona em ambientes dinâmicos, modificando a força de repulsão axial de obstáculos para forças de repulsão circulares. Essa mudança também elimina a possibilidade de as aeronaves ficarem presas em mínimos locais. Além disso, a estratégia proposta torna os caminhos gerados mais eficientes para prevenção de colisão de obstáculos dinâmicos do que outras estratégias de campos de força. Entretanto, a estratégia foi aplicada em um cenário com apenas três drones e em duas dimensões, deixando a extensão 
para três dimensões nos trabalhos futuros. O artigo [Sun et al. 2017] também propõe uma estratégia baseada em campos de força para drones em cooperação em ambiente 3D dinâmico, considerando que, na prática, a distância de segurança de um drone é muito maior do que seu volume. Cada trajetória é planejada considerando que apenas os drones em colaboração são obstáculos dinâmicos e que os drones viajam apenas no eixo horizontal, o que são limitações do trabalho. Os autores simularam apenas seis drones e 30 obstáculos nos eixos horizontal e vertical para simular prédios e outras construções em cidades.

O trabalho [Hrabar 2011] utiliza uma estratégia de detecção e prevenção que considera um cilindro de segurança ao longo do sentido do drone. Obstáculos que entrem nesse cilindro são identificados como colisores em potencial e o algoritmo busca por um ponto de escape ao longo de elipses que se expandem a partir do centro do cilindro de segurança, de forma que o ponto de escape escolhido evite colisões tanto com o obstáculo mais próximo quanto com outros obstáculos no ambiente. O ponto de escape escolhido ainda aponta para o próximo destino do drone e, assim, essa estratégia pode ser utilizada como solução única em algumas situações ou pode ser integrada com uma estratégia deliberativa. Entretanto, os resultados apresentados foram gerados com ambientes estáticos, embora os autores considerem que a abordagem também funciona em ambientes dinâmicos.

Por fim, o trabalho [Gageik et al. 2015] adota uma estratégia de detecção e prevenção com três classificações de regiões dentro do alcance dos sensores. A região mais próxima ao drone é a região de perigo, onde uma decisão para evitar colisão deve ser tomada imediatamente quando um obstáculo se encontra nela. A região intermediária é chamada de região próxima, em que um obstáculo presente nela deve ser monitorado e o drone deve controlar sua distância até esse obstáculo. A região mais afastada é a região livre, onde o drone não toma decisões mesmo na presença de obstáculos. Os autores também consideram a possibilidade de os drones estarem entre dois obstáculos e, nessa situação, o drone tenta se manter na posição central entre eles. Contudo, essa estratégia não considera colisões que podem acontecer no eixo vertical devidas a drones que modificam sua altura, como em pousos e decolagens.

Os trabalhos avaliados em nossa revisão bibliográfica mostram que a comunidade têm interesse no campo de prevenção de colisões e ele é bastante explorado, com diversos tipos de estratégias. Contudo, a maioria dos trabalhos avaliou cenários com poucos drones e em duas dimensões, enquanto o cenário de serviço de entregas ocorre em três dimensões e pode conter um grande número de drones em operação. Para este trabalho, consideramos um cenário em três dimensões em que os drones não desviam para termos um patamar inicial do número de colisões para comparação, uma estratégia de detecção e prevenção ingênua em que os drones tomam um desvio aleatório para tentar evitar uma possível colisão e uma estratégia de detecção e prevenção inspirada pela aviação, em que os drones tomam um desvio à direita.

\subsection{Simuladores de Drones}

Os artigos [Craighead et al. 2007, Hentati et al. 2018, Mairaj et al. 2019] revisam simuladores comerciais e de código aberto de veículos não tripulados, simuladores para análise de desempenho em sistemas de drones e simuladores de drones para aplicações es- 
pecíficas, respectivamente. Simuladores como X-Plane, FightGear, Microsoft Flight Simulator, UE4Sim e AirSim possuem foco no controle de navegação do drone e em um drone único ou poucos drones. Para o treinamento de pilotos, os simuladores Zephyr Sim, droneSim Pro-, DRL Simulator, Real Drone Simulator, RealFlight Simulator, VAMPIRE Suite, Heli-X, além do Microsoft Flight Simulator são utilizados, assim como para entretenimento. Os simuladores JMavSim, Simbeeotic, DIMAV e RotorS foram projetados para simulação de microveículos aéreos. Além disso, o Simbeeotic e os simuladores SUAAVE e RAVEN focam na simulação de enxames de drones que viajam em grupo. Por fim, o simulador RotorS também pode ser utilizado para planejamento de rotas.

AirSim é o simulador de código aberto da Microsoft para drones e carros desenvolvidos com Unreal Engine, que é uma plataforma de criação 3D que inclui simulações físicas e visuais realistas [Shah et al. 2018]. Suas principais características são a possibilidade de controlar veículos com software-and-hardware-in-the-loop, simular cenários realistas e capturar imagens para algoritmos de aprendizado de máquina profundo, visão computacional e aprendizado por reforço para veículos autônomos. Como desvantagens, podemos citar o foco em apenas um drone e nas aplicações citadas e a falta de uma resposta completa à colisão.

Gazebo é uma plataforma de simulação 3D para robótica que simula a física dos objetos e possui um conjunto de modelos de sensores [Open Source Robotics Foundation 2021]. Contudo, ela não possui código específico para simulação de drones, sendo necessário construir um simulador de drones e modelar todos aspectos necessários para a simulação dos drones desde o início.

UTSim é um simulador de drones de código aberto desenvolvido em C\# com a plataforma Unity [Al-Mousa et al. 2019]. Ele pode simular mais de 1500 drones, suas especificações físicas e as variadas questões sobre a integração do tráfego aéreo, como navegação, controle, protocolos de comunicação e detecção e prevenção de colisões com objetos estáticos ou dinâmicos. O simulador permite utilizar qualquer ambiente aceito pela Unity, como cidades com prédios de diferentes alturas, que podem impor obstáculos aos drones, e diferentes condições climáticas, como chuvas ou rajadas de vento, que podem inserir perturbações nas trajetórias dos drones. O simulador também permite definir drones com diferentes características e utilizar diferentes algoritmos para detecção e prevenção de colisões. Cada drone tem sua trajetória controlada por um controlador do tipo proporcional-integral-derivativo (PID). Escolhemos esse simulador para desenvolver nosso trabalho devido à sua facilidade de utilização, modificação, suas características apropriadas para simulação de um serviço de entregas por drones em cidades inteligentes e a ele ser um simulador já referenciado na literatura [Olawale et al. 2020, Carramiñana et al. 2021, Grigoropoulos and Lalis 2020].

\section{Cenário Proposto}

O cenário da Figura 1 ilustra um serviço de entregas aéreas de encomendas utilizando drones, considerando duas operadoras de logística de entregas (laranja e azul), além de drones que cumprem outras missões não identificadas (cinza). Existem dois centros de distribuição (CD) de mercadorias para as operadoras laranja e azul, onde decolagens para iniciar entregas e pousos no retorno ao CD ocorrem em dronepontos (identificados pela letra D). Além dos dronepontos dos CDs das operadoras logísticas, existem drone- 
pontos menores próximos aos locais de entrega. Um cenário real pode incluir diversas operadoras de serviços de entregas com drones [Frachtenberg 2019], drones cumprindo missões esporádicas [Ayamga et al. 2021], além de drones para transporte de pessoas [Kasliwal et al. 2019].

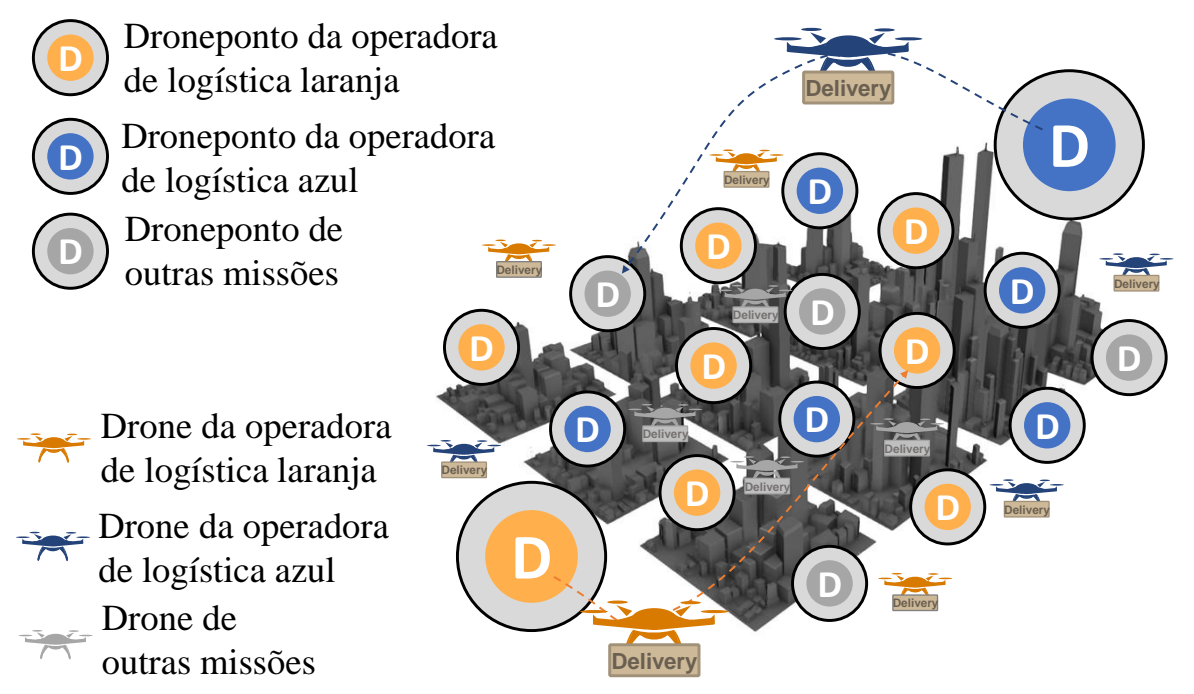

Figura 1. Cenário de Serviço de Entregas baseado em Drones

O principal desafio de um serviço de entregas baseado em drones é evitar colisões [Yang and Wei 2021], que podem envolver outros drones, objetos voadores, pássaros, ou obstáculos como prédios e árvores. De forma semelhante a aeronaves, os maiores riscos se concentram próximos aos dronepontos em pousos e decolagens [Kleinbekman et al. 2018]. No entanto, ao contrário do sistema centralizado de controle de tráfego aéreo, um cenário de entregas envolvendo várias operadoras logísticas não possui coordenação centralizada global. Por esse motivo, as operadoras podem implementar sistemas otimizados de sequenciamento de pousos e decolagens nos dronepontos dos seus CDs. Porém, os drones devem também ter a capacidade de evitar colisões no ar, ou seja, fora dos pontos de pouso e decolagem, seja no CD da operadora, ou nos dronepontos de entrega.

A Figura 2 ilustra quatro situações típicas onde colisões entre drones podem ocorrer. Essas situações podem ocorrer com frequência em um cenário de entregas se nenhum drone tomar atitudes para evitar colisões, mesmo em um cenário com poucos drones. $\mathrm{Na}$ Figura 2a um drone decolando do droneponto está em rota de colisão com um drone que está passando pela região e na Figura 2 b ocorre o contrário, ou seja, um drone pousando no droneponto está em rota de colisão com outro drone. Em ambas as figuras, os drones são de operadoras diferentes, porque uma mesma operadora poderia evitar tais situações através de um controle centralizado. Mesmo entre operadoras diferentes, seria possível evitar tais colisões através da instituição de regiões proibidas, como são os aeroportos na aviação. A Figura $2 \mathrm{c}$ representa uma situação em que drones de uma mesma operadora podem colidir enquanto decolam e pousam, por falta de um controle de sequenciamento de uso do droneponto. Finalmente, o cenário que requer abordagens mais sofisticadas para evasão de colisões é representado pela Figura 2d, onde drones de operadoras diferentes estão voando em rota de colisão. 


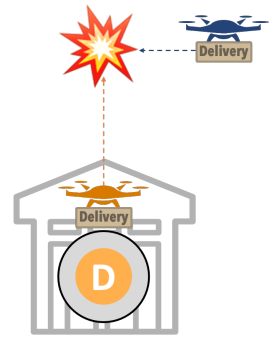

(a) Decolagem: operadoras diferentes

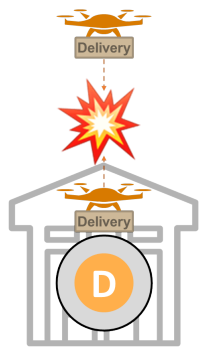

(c) Pouso e decolagem: mesma operadora

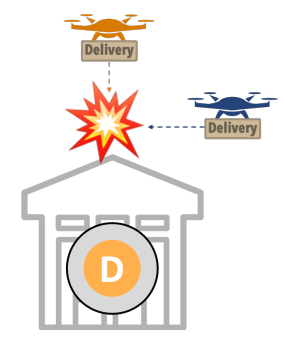

(b) Pouso: operadoras diferentes

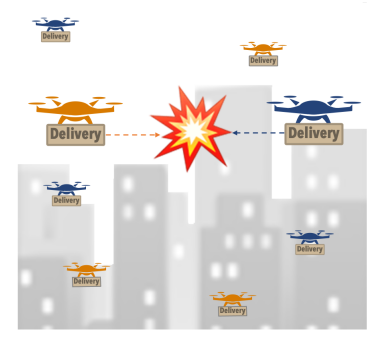

(d) Cruzeiro: operadoras diferentes

Figura 2. Colisões entre drones: diferentes possibilidades

\section{Metodologia}

As simulações foram executadas usando nossa versão modificada do simulador UTSim. Para simular o cenário proposto, dispusemos os dronepontos que representam os CDs ao longo de um quadrado de $1 \mathrm{~km}$ de lado, a cada $40 \mathrm{~m}$, conforme mostra a Figura 3. Os dronepontos dos CDs estão representados por círculos laranjas. Os drones realizam entregas dentro da região delimitada por um quadrado de $900 \mathrm{~m}$, de forma que nenhuma entrega acontece em distâncias menores do que $50 \mathrm{~m}$ de qualquer droneponto. A região amarela delimita uma área próxima aos dronepontos onde consideramos qualquer colisão que aconteça nela uma colisão devida aos pousos e decolagens de drones. Qualquer ponto dessa região encontra-se ao menos $50 \mathrm{~m}$ distante dos pontos de entrega e do restante da cidade, ou seja, dos pontos externos ao quadrado de $1 \mathrm{~km}$ onde estão os dronepontos.

Cada drone mede $2 \mathrm{~m} \times 2 \mathrm{~m} \times 0,5 \mathrm{~m}$ e possui um raio de colisão de $20 \mathrm{~m}$. Sempre que um objeto, seja um drone ou um obstáculo, entra no raio de colisão de um drone, ele pode tomar alguma atitude para evitar uma possível colisão. Utilizamos seis frotas de tamanhos diferentes para simular o cenário com 2, 5, 10, 20, 50 e 100 drones, que decolam a cada $10 \mathrm{~s}$ de um droneponto aleatório para mitigar colisões que podem acontecer próximas aos dronepontos devidas às decolagens e aos pousos. Após a definição do droneponto, os drones executam uma sequência de 10 comandos: 1) inicialização, 2) definição de velocidade de $20 \mathrm{~m} / \mathrm{s}, 3$ ) decolagem até altura de $10 \mathrm{~m}, 4)$ viagem até destino, 5) pouso até altura de $1 \mathrm{~m}, 6$ ) espera de $10 \mathrm{~s}$ para ocorrer a entrega, 7) decolagem até altura de $10 \mathrm{~m}, 8)$ retorno até droneponto inicial, 9) pouso até altura de $1 \mathrm{~m} \mathrm{e} \mathrm{10)} \mathrm{inativação.}$ Nas abordagens com desvios, os drones podem incluir destinos temporários nesta lista de comandos para tentar evitar uma colisão.

Utilizamos três abordagens para comparação. Primeiro, quando um drone entra no raio de colisão de outro drone, ele não toma atitude alguma e continua a seguir seu destino; chamamos essa abordagem de fazer nada. Na segunda abordagem, o drone que 


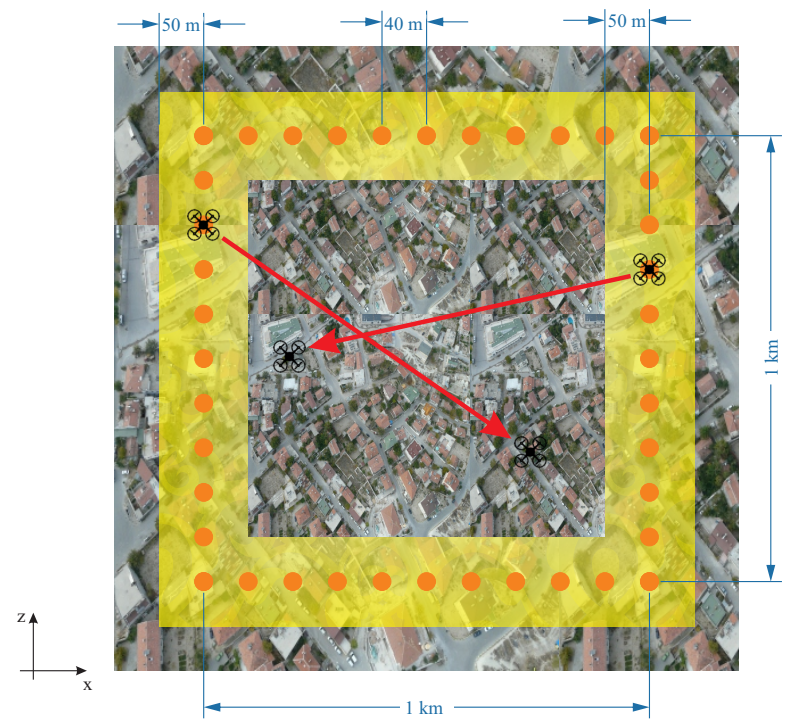

Figura 3. Vista superior da configuração utilizada para simular o cenário proposto [Braxmeier 2021] (modificada).

entra no raio de colisão de outro drone toma um desvio aleatório entre - $40 \mathrm{~m}$ e $40 \mathrm{~m}$ em relação a sua posição nos eixos x e z, ou seja, entre $-56,6 \mathrm{~m}$ e 56,6 m, sem variação de altura. Por fim, na terceira abordagem, um drone que entra no raio de colisão de outro drone toma um desvio de $20 \mathrm{~m}$ em relação a sua posição nos eixos x e z, ou seja, $28,3 \mathrm{~m}$, à direita em relação ao seu sentido, novamente sem variar a altura. Enquanto nós utilizamos a primeira abordagem (fazer nada) para conhecer as condições de colisão do cenário, a abordagem de tomar um desvio aleatório representou uma solução ingênua e a abordagem de tomar um desvio à direita foi inspirada na prevenção de colisão na aviação. Executamos as simulações 30 vezes para cada tamanho de frota e abordagem e reportamos a média e o intervalo de confiança de $95 \%$ para cada resultado.

\section{Resultados e Discussão}

A Figura 4 mostra a média e o desvio padrão do número de drones que colidiram para cada configuração de frota. Quando temos poucos drones no cenário, como dois ou cinco drones, as abordagens que tomam desvios são capazes de reduzir ou eliminar o número de colisões, com o desvio à direita sendo até $20 \%$ melhor do que o desvio aleatório, o que seria esperado de uma abordagem inspirada na aviação. Contudo, a partir de 10 drones no cenário, as abordagens com desvios mantêm ou aumentam o número de drones que colidem em média, sendo que, para 50 e 100 drones, o número de colisões aumenta em até $50 \%$ para o desvio aleatório e $25 \%$ para o desvio à direita. Considerando que, na aviação, não se espera um grande número de aeronaves em rota de colisão devido à baixa densidade de aeronaves e à utilização de aerovias, uma abordagem que funciona nesse caso pode não funcionar para um cenário com alta densidade de drones e sem aerovias.

A Figura 5 mostra a porcentagem de colisões que ocorreram pelo menos $50 \mathrm{~m}$ distantes de qualquer droneponto, ou seja, colisões que não ocorreram devido à tentativa de decolagem ou pouso em um droneponto do CD. Na abordagem fazer nada, quando há poucos drones na frota, a maioria das colisões ocorrem próximas aos dronepontos, enquanto que, com um número maior de drones, o número de colisões que ocorrem durante 


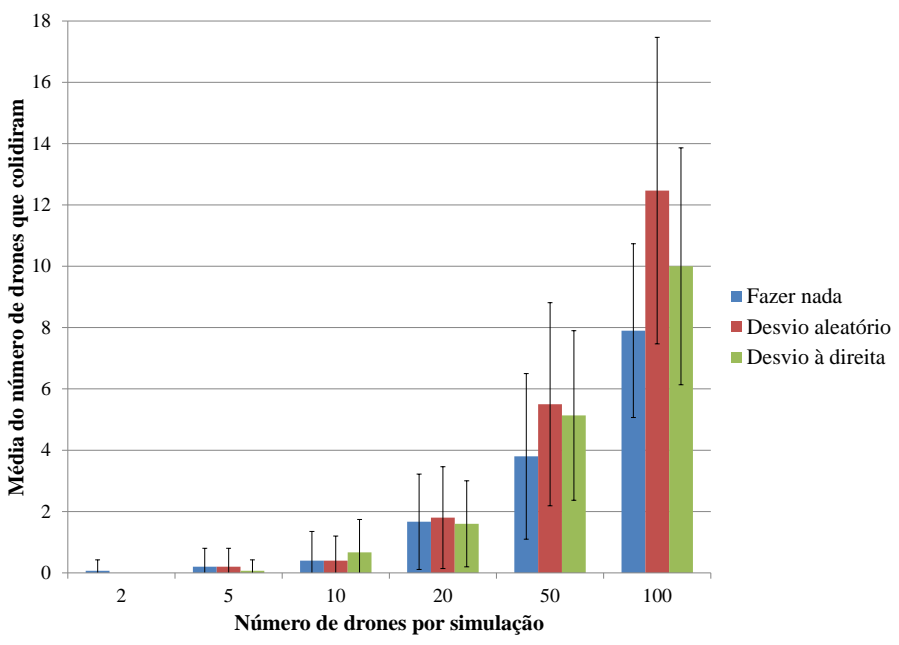

Figura 4. Média e desvio padrão do número de drones que colidiram em $\mathbf{3 0}$ simulações.

o cruzeiro predominam. Já nas abordagens com desvios, pelo menos $80 \%$ das colisões ocorrem longe dos dronepontos, o que, em comparação com a abordagem fazer nada, indica que os desvios são capazes de evitar colisões relacionadas à tentativa de decolagem e pouso num primeiro momento, mas acabam fazendo os drones colidirem em um ponto mais distante da região dos dronepontos. Essa figura também ilustra que existem dois tipos de problemas no cenário de entregas com drones. Um problema é a colisão próxima aos dronepontos dos CDs relativa à tentativa de decolagem e pouso, e o outro problema é a colisão que ocorre durante a entrega, podendo ser durante a viagem ou durante a tentativa de decolagem e pouso para a entrega em si.

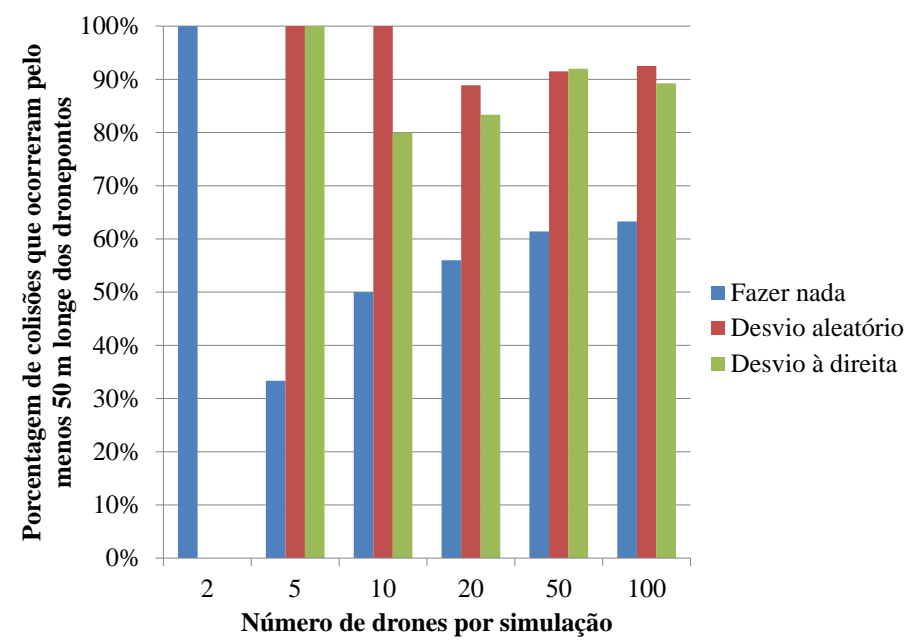

Figura 5. Porcentagem do número de drones que colidiram pelo menos $50 \mathrm{~m}$ longe dos dronepontos em 30 simulações.

A Figura 6 mostra o tempo médio decorrido até o drone colidir e o intervalo de confiança de $95 \%$. Com exceção das frotas menores, o tempo médio até colisão dos drones que tomam desvios é até duas vezes maior do que o tempo médio de colisão dos drones que não tomam desvios. Os resultados dessa figura indicam que, inicialmente, nas 
abordagens que tomam desvios, quando um drone entra no raio de colisão de outro drone, ambos os drones conseguem tomar decisões que evitam uma possível colisão. Entretanto, eles não conseguem sair da situação de colisão, seja por tentarem voltar ao mesmo ponto onde o drone entrou no raio de colisão do outro, seja por entrarem no raio de colisão de outros drones, eventualmente colidindo por tomarem um desvio prejudicial que não considera a presença de vários drones na proximidade.

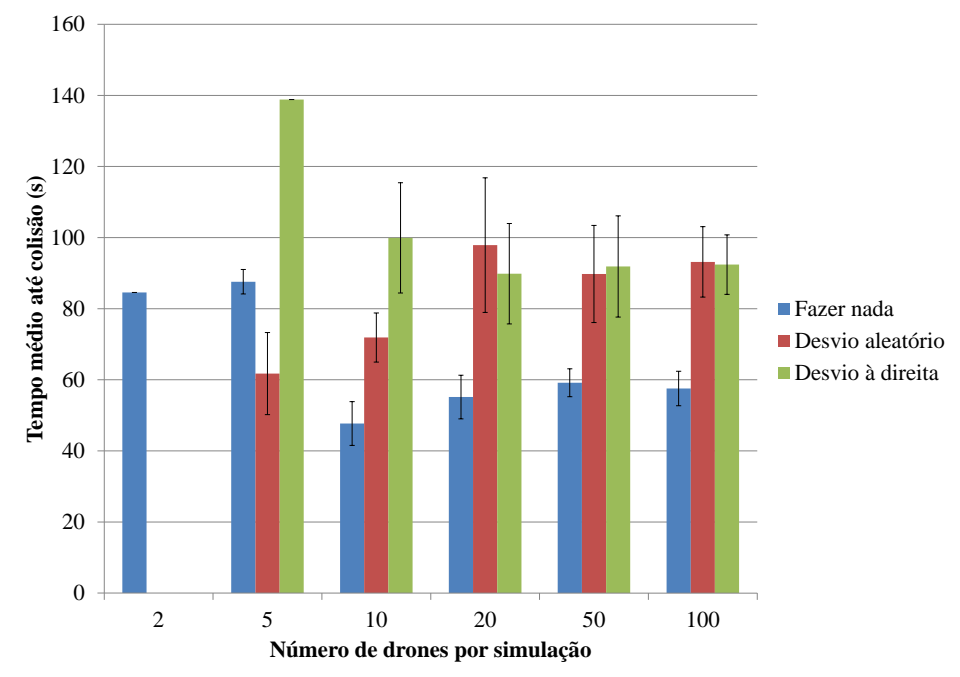

Figura 6. Média e intervalo de confiança de $95 \%$ do tempo até colisão em 30 simulações.

A Figura 7 mostra o tempo médio das viagens que tiveram sucesso, ou seja, dos drones que fizeram a entrega e voltaram ao $\mathrm{CD}$ de origem. Na maioria das configurações de frota, o tempo médio das viagens com sucesso para drones que tomam desvios é até $23,6 \%$ maior do que o tempo para os drones que não tomam desvios. Esse resultado indica que, quando os desvios são capazes de impedir colisões, eles aumentam o tempo de viagem razoavelmente, o que é ineficiente e impacta o tempo de bateria dos drones, podendo impedir que o drone realize a entrega e retorne ao CD. Além disso, considerando que, para a frota de 100 drones, a cada momento da simulação, podemos ter até 11 drones na abordagem fazer nada e até 14 drones nas abordagens com desvios, ao todo uma área de $44 \mathrm{~m}^{2}$ e $56 \mathrm{~m}^{2}$ pode ser ocupada pelos drones na região de entrega, respectivamente. Essa área representa entre $5 \%$ e $6 \%$ da região de entrega, indicando que podemos aumentar a densidade de drones nesse cenário para mais de 14 drones $/ \mathrm{km}^{2}$.

Por fim, a Figura 8 mostra o tempo médio de entrega, que compreende o tempo decorrido entre pouso, entrega em si e decolagem até a altura de cruzeiro. As abordagens com desvios tiveram um tempo de entrega até $8 \%$ maior do que a abordagem sem desvios. Como a diferença entre a altura de entrega e a altura de cruzeiro é $9 \mathrm{~m}$ e o raio de colisão é $20 \mathrm{~m}$, quando um drone sobrevoa a região de entrega de outro drone, mesmo que eles não estejam em rota de colisão, nas abordagens com desvios, ambos os drones tomam desvios, o que atrasa sem motivos tanto o drone que está em cruzeiro quanto o drone que está realizando a entrega. Além do tempo médio de entrega, o tempo médio de decolagem foi de 7,5 s e o tempo médio de cruzeiro, incluindo a viagem de ida e a viagem de volta, foi de $71 \mathrm{~s}$ para a abordagem fazer nada, $89 \mathrm{~s}$ para o desvio aleatório e $92 \mathrm{~s}$ para o desvio à direita. $\mathrm{O}$ tempo médio de pouso foi 7,2 s para todas as abordagens e somente um drone 


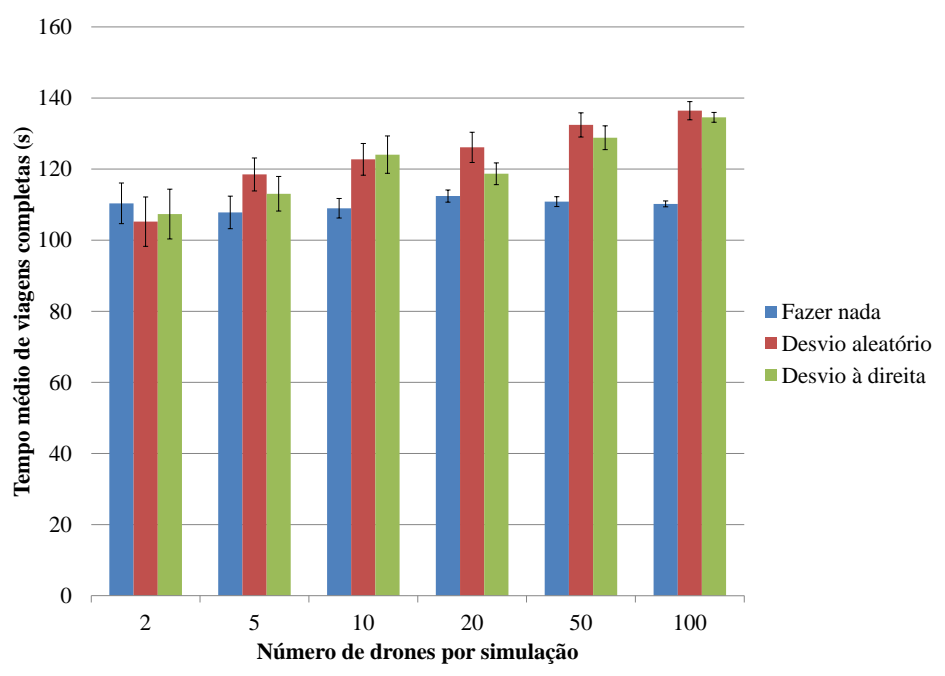

Figura 7. Média e intervalo de confiança de $95 \%$ do tempo de viagens completas em 30 simulações.

foi prejudicado durante o pouso no CD pela aproximação de outro drone. Esse resultado era esperado devido ao desenho das simulações com drones pousando em e decolando de diferentes dronepontos escolhidos aleatoriamente.

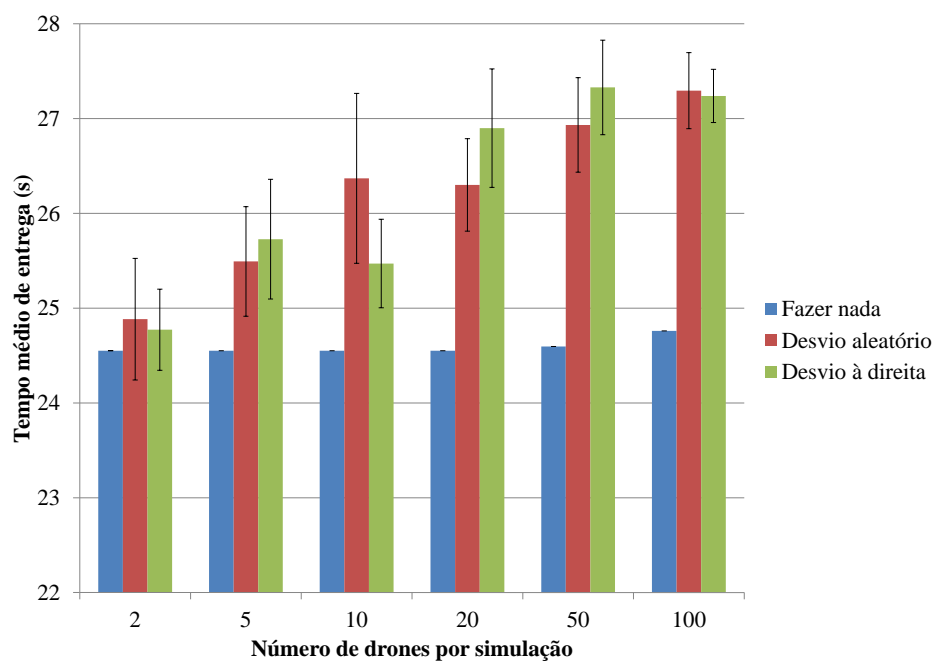

Figura 8. Média e intervalo de confiança de $95 \%$ do tempo de entrega em 30 simulações.

\section{Limitações do Trabalho}

As limitações do nosso trabalho podem ser divididas em quatro pontos. O primeiro ponto refere-se ao tamanho do raio de colisão, que pode interferir no número de colisões nas estratégias com desvios. Quando se aumenta o raio de colisão, o drone pode identificar um obstáculo a uma distância maior e, assim, há um espaço de manobra maior para evitar uma colisão. Considerando o alcance de sensores comuns, como o sonar e o LiDAR, é possível aumentar esse raio para até $400 \mathrm{~m}$, embora as estratégias propostas neste artigo ficariam mais ineficientes ao tomar desvios a uma distância tão grande dos obstáculos. 
Entretanto, as abordagens podem ser melhoradas para considerar o alcance dos sensores e tomar decisões mais adequadas. De fato, já estamos conduzindo simulações para avaliar o efeito do tamanho do raio de colisão e o tamanho do desvio tomado no número de colisões. Ambas as estratégias apresentadas neste artigo tiveram um número de colisões igual ou maior quando o tamanho do desvio foi reduzido para até 7 metros, evidenciando que os tamanhos de desvio utilizados neste artigo devem ser mantidos ou aumentados quando o raio de colisão for alterado.

O segundo ponto é que podemos aumentar o número de drones no cenário proposto para avaliar o comportamento de todas as medidas apresentadas. Como terceiro ponto, há de se considerar a simplicidade das estratégias apresentadas em relação às estratégias da literatura e mesmo a uma estratégia de sucesso na aviação civil, que, nos resultados apresentados, não foram capazes de reduzir o número de colisões nas frotas com maior número de drones. Além disso, essas estratégias precisam ser comparadas a estratégias de prevenção de colisão mais sofisticadas. Por fim, o quarto ponto é falta da relação entre uma colisão e a etapa da viagem que o drone estava quando aconteceu essa colisão, o que pode trazer novas informações sobre o cenário proposto. Todas essas limitações serão consideradas nos trabalhos futuros.

\section{Conclusão e Trabalhos Futuros}

Neste trabalho, propusemos um cenário de serviço de entregas por drones em cidades inteligentes e avaliamos o número de drones que colidem sob três abordagens diferentes. Avaliamos o cenário com frotas de drones crescentes, variando de 2 a 100 drones, e três abordagens quando um drone entra no raio de colisão de outro drone, ou seja, quando uma colisão é detectada: fazer nada, quando os drones não tomam desvios, desvio aleatório e desvio à direita igual ao raio de colisão nos eixos x e $\mathrm{z}$.

Os resultados mostraram que, embora para uma frota pequena com dois ou cinco drones o número de colisões para as abordagens com desvios tenha sido igual ou menor do que fazer nada, para frotas maiores, as abordagens com desvios aumentaram o número de colisões. Considerando que a abordagem de tomar um desvio à direita foi inspirada pela prevenção de colisões na aviação, nossos resultados corroboram que ela é uma abordagem efetiva na prevenção de colisão com poucos veículos aéreos, contudo, ela não impede colisões em um cénário com densidade maior de drones.

Também mostramos que existem dois tipos de problemas relevantes nesse cenário: colisões que podem ocorrer em tempo de cruzeiro e colisões que podem ocorrer devido à tentativa de decolagem e/ou pouso no mesmo droneponto ou em dronepontos muito próximos. Os resultados com os tempos médios até colisão e até o fim da viagem com sucesso mostraram que as abordagens com desvios são ineficientes, aumentando em até $23,6 \%$ o tempo de uma viagem com sucesso. Já o tempo médio de entrega revelou que drones em cruzeiro sobrevoando um droneponto de entrega podem interferir em um drone que está realizando uma entrega, mesmo que não haja colisão possível entre eles. Por fim, nossos resultados mostraram que é possível aumentar a densidade de drones para mais de 14 drones $/ \mathrm{km}^{2}$.

Como trabalhos futuros, aumentaremos essa densidade de drones, avaliaremos o efeito do tamanho do raio de colisão no número de colisões, controlaremos de forma centralizada os pousos e decolagens em cada droneponto, utilizaremos abordagens so- 
fisticadas de prevenção de colisão, como uma abordagem local de detecção e prevenção que tome diferentes decisões de acordo com a distância do obstáculo e leve em conta a direção e sentido de todos os drones, e compararemos essas abordagens sofisticadas com as estratégias propostas neste artigo. Como abordagens locais não evitam colisões em todos os casos, posteriormente, pretendemos integrar essa abordagem local a uma abordagem híbrida que também utilize uma abordagem global. Para melhoria do simulador, implementaremos a adição de cargas aos drones, a consideração do gasto energético dos vôos, pesos das cargas e algoritmos executados nos drones em suas baterias e o poder de processamento e comunicação no tempo de resposta dos algoritmos de prevenção de colisão.

\section{Agradecimentos}

Esta pesquisa é parte do INCT da Internet do Futuro para Cidades Inteligentes, financiado por CNPq (proc. 465446/2014-0), Coordenação de Aperfeiçoamento de Pessoal de Nível Superior - Brasil (CAPES) - Código de Financiamento 001 e FAPESP (procs. 14/50937$1,15 / 24485-9$ e 20/05152-7) e da UFABC.

\section{Referências}

Al-Mousa, A., Sababha, B. H., Al-Madi, N., Barghouthi, A., and Younisse, R. (2019). UTSim: A framework and simulator for UAV air traffic integration, control, and communication. International Journal of Advanced Robotic Systems, 16(5):1729881419870937.

Ayamga, M., Akaba, S., and Nyaaba, A. A. (2021). Multifaceted applicability of drones: A review. Technological Forecasting and Social Change, 167:120677.

Braxmeier, H. (2021). Göreme, Capadócia, Turquia. https://

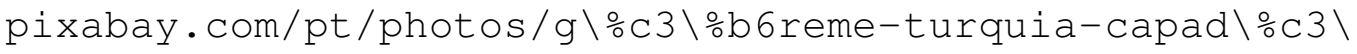
을ia-casa-64874/. Acessado em 9 de julho de 2021.

Carramiñana, D., Campaña, I., Bergesio, L., Bernardos, A. M., and Besada, J. A. (2021). Sensors and Communication Simulation for Unmanned Traffic Management. Sensors, 21(3).

Choi, D. and Kim, D. (2020). Enhanced Potential Field-Based Collision Avoidance for Unmanned Aerial Vehicles in a Dynamic Environment. Journal of Intelligent \& Robotic Systems, pages 1-7.

Craighead, J., Murphy, R., Burke, J., and Goldiez, B. (2007). A survey of commercial open source unmanned vehicle simulators. In Proceedings 2007 IEEE International Conference on Robotics and Automation, pages 852-857.

Frachtenberg, E. (2019). Practical drone delivery. Computer, 52(12):53-57.

Gageik, N., Benz, P., and Montenegro, S. (2015). Obstacle Detection and Collision Avoidance for a UAV With Complementary Low-Cost Sensors. IEEE Access, 3:599-609.

Grigoropoulos, N. and Lalis, S. (2020). Simulation and digital twin support for managed drone applications. In 2020 IEEE/ACM 24th International Symposium on Distributed Simulation and Real Time Applications (DS-RT), pages 1-8.

Guan, X., Lyu, R., Shi, H., and Chen, J. (2020). A survey of safety separation management and collision avoidance approaches of civil UAS operating in integration national 
airspace system. Chinese Journal of Aeronautics, 33(11):2851-2863. SI: Emerging Technologies of Unmanned Aerial Vehicles.

Hentati, A. I., Krichen, L., Fourati, M., and Fourati, L. C. (2018). Simulation tools, environments and frameworks for uav systems performance analysis. In 2018 14th International Wireless Communications Mobile Computing Conference (IWCMC), pages 1495-1500.

Hrabar, S. (2011). Reactive obstacle avoidance for Rotorcraft UAVs. In 2011 IEEE/RSJ International Conference on Intelligent Robots and Systems, pages 4967-4974.

Kasliwal, A., Furbush, N. J., Gawron, J. H., McBride, J. R., Wallington, T. J., De Kleine, R. D., Kim, H. C., and Keoleian, G. A. (2019). Role of flying cars in sustainable mobility. Nature communications, 10(1):1-9.

Kleinbekman, I. C., Mitici, M. A., and Wei, P. (2018). eVTOL arrival sequencing and scheduling for on-demand urban air mobility. In 2018 IEEE/AIAA 37th Digital Avionics Systems Conference (DASC), pages 1-7. IEEE.

Lin, Z., Castano, L., Mortimer, E., and Xu, H. (2020). Fast 3D Collision Avoidance Algorithm for Fixed Wing UAS. Journal of Intelligent \& Robotic Systems, 97:577604.

Mairaj, A., Baba, A. I., and Javaid, A. Y. (2019). Application specific drone simulators: Recent advances and challenges. Simulation Modelling Practice and Theory, 94:100117.

Olawale, O. P., Dimililer, K., and Al-Turjman, F. (2020). Chapter Six - AI simulations and programming environments for drones: an overview. In Al-Turjman, F., editor, Drones in Smart-Cities, pages 93-106. Elsevier.

Open Source Robotics Foundation (2021). Gazebo. http://gazebosim.org/. Acessado em 11 de julho de 2021.

Seo, J., Kim, Y., Kim, S., and Tsourdos, A. (2017). Collision Avoidance Strategies for Unmanned Aerial Vehicles in Formation Flight. IEEE Transactions on Aerospace and Electronic Systems, 53(6):2718-2734.

Shah, S., Dey, D., Lovett, C., and Kapoor, A. (2018). Airsim: High-fidelity visual and physical simulation for autonomous vehicles. In Hutter, M. and Siegwart, R., editors, Field and Service Robotics, pages 621-635, Cham. Springer International Publishing.

Sánchez, P., Casado, R., and Bermúdez, A. (2020). Real-Time Collision-Free Navigation of Multiple UAVs Based on Bounding Boxes. Electronics, 9(10).

Sun, J., Tang, J., and Lao, S. (2017). Collision Avoidance for Cooperative UAVs With Optimized Artificial Potential Field Algorithm. IEEE Access, 5:18382-18390.

Yang, X. and Wei, P. (2021). Autonomous free flight operations in urban air mobility with computational guidance and collision avoidance. IEEE Transactions on Intelligent Transportation Systems.

Yasin, J. N., Mohamed, S. A. S., Haghbayan, M.-H., Heikkonen, J., Tenhunen, H., and Plosila, J. (2020). Unmanned Aerial Vehicles (UAVs): Collision Avoidance Systems and Approaches. IEEE Access, 8:105139-105155. 